\title{
Photonic two-qubit parity gate with tiny cross-Kerr nonlinearity
}

\author{
Xin-Wen Wang,,${ }^{1,2, *}$ Deng-Yu Zhang, ${ }^{1, \dagger}$ Shi-Qing Tang, ${ }^{1}$ Li-Jun Xie, ${ }^{1}$ Zhi-Yong Wang, ${ }^{3 \ddagger}$ and Le-Man Kuang ${ }^{2, \S}$ \\ ${ }^{1}$ Department of Physics and Electronic Information Science, \\ Hengyang Normal University, Hengyang 421008, People's Republic of China \\ ${ }^{2}$ Key Laboratory of Low-Dimensional Quantum Structures and Quantum Control of Ministry of Education, \\ and Department of Physics, Hunan Normal University, Changsha 410081, People's Republic of China \\ ${ }^{3}$ School of Optoelectronic Information, Chongqing University of Technology, Chongqing 400054, People's Republic of China
}

\begin{abstract}
The cross-Kerr nonlinearity (XKNL) effect can induce efficient photon interactions in principle with which photonic multiqubit gates can be performed using far fewer physical resources than linear optical schemes. Unfortunately, it is extremely challenging to generate giant cross-Kerr nonlinearities. In recent years, much effort has been made to perform multiqubit gates via weak XKNLs. However, the required nonlinearity strengths are still difficult to achieve in the experiment. We here propose an XKNL-based scheme for realizing a twophoton polarization-parity gate, a universal two-qubit gate, in which the required strength of the nonlinearity could be orders of magnitude weaker than those required for previous schemes. The scheme utilizes a ring cavity fed by a coherent state as a quantum information bus which interacts with a path mode of the two polarized photons (qubits). The XKNL effect makes the bus pick up a phase shift dependent on the photon number of the path mode. Even when the potential phase shifts are very small they can be effectively measured using photon-number resolving detectors, which accounts for the fact that our scheme can work in the regime of tiny XKNL. The measurement outcome reveals the parity (even parity or odd parity) of the two polarization qubits.
\end{abstract}

PACS numbers: 03.67.Lx, 42.50.Ex, 42.50.Dv

Keywords: Parity gate, photonic qubit, tiny cross-Kerr nonlinearity

\section{INTRODUCTION}

The optical system is among the most popular physical systems for implementing quantum computation. This is mainly due to the facts that light quantum states are generally more robust against decoherence than most massive qubit systems, and that the computation programs can be implemented by simple optical elements plus photodetections. In addition, all-optical quantum computation can be combined with quantum communication without qubit interconversion. In all-optical quantum information processing (QIP), the qubits are usually encoded by singlephoton polarization states (other types of photonic qubits can be usually converted to polarization qubits by optical apparatuses). Parametric down-conversion can produce polarization-entangled photon pairs and heralded single photons. Beam splitters (BSs) and wave plates can be used to accomplish arbitrary single-qubit rotations. To complete a universal gate set for quantum computation, the key point that is then needed is an appropriate two-qubit quantum gate, such as a two-qubit parity gate [1, 2] from which a controlled-NOT (CNOT) gate can be readily constructed [3-5]. Note that a universal gate set can also serve many quantum communication protocols, in that it can be utilized to implement their required entangled-state joint measurements and entangled-channel generations.

To implement a photonic two-qubit gate, nonlinear interactions between individual photons are required. Linear optical elements plus photodetections can induce effective nonlinear photon interactions in principle. This way, however, is nondeterministic, and needs consuming substantial ancillary photon resources for achieving a high efficiency [6-10], which is the main obstacle to large-scale

\footnotetext{
*xwwang@mail.bnu.edu.cn

†dyzhang672@163.com

¥wzyong@cqut.edu.cn

$\S$ lmkuang@hunnu.edu.cn
}

QIP with linear optics. The required optical nonlinearity can also be achieved directly using a cross-Kerr medium that can be described by an interaction Hamiltonian of the form $H=-\hbar \chi a_{p}^{+} a_{p} a_{s}^{+} a_{s}[8,11]$. Here $a_{p}\left(a_{p}^{+}\right)$and $a_{s}$ $\left(a_{s}^{+}\right)$are, respectively, the annihilation (creation) operators of modes $p$ and $s$, and $\chi$ is the strength of the nonlinearity. Transforming the mode $p(s)$ using this Hamiltonian will induce a phase shift that depends on the number of photons in the mode $s(p)$. Indeed, the mode transformations of the two beams are $a_{p} \rightarrow a_{p} \exp \left(i \theta a_{s}^{+} a_{s}\right)$ and $a_{s} \rightarrow a_{s} \exp \left(i \theta a_{p}^{+} a_{p}\right)$, where $\theta=\chi t$ with $t$ being the interaction time. When $\theta=\pi$, a two-photon controlledphase gate is naturally implemented, from which a CNOT gate can also be easily constructed. With the giant crossKerr phase shift (XKPS), many schemes for realizing optical quantum nondemolition (QND) measurements, photonic quantum gates, optical entangled states, and quantum communication protocols have been proposed (see, e.g., [11-17]).

Unfortunately, even the largest natural XKNL is extremely weak. Operating in the single-photon regime with a mode volume of about $0.1 \mathrm{~cm}^{3}$, the XKPS is only $\theta \approx$ $10^{-18}$ [18]. This makes cross-Kerr-based optical quantum gates and QIP extremely challenging. To obtain a giant XKPS, one can in principle lengthen the cross-Kerr interaction time by manufacturing a long fiber with the crossKerr materials [19]. In this case, however, photon losses and self-phase modulation in the cross-Kerr medium will prevent the gate from operating properly [20, 21]]. Since the end of last century, much effort has been made to generate larger XKNLs using electromagnetically induced transparency (EIT) (see, e.g., [22-30]). Although considerable progress has been made, the experimentally available XKNL still cannot satisfy the requirement [31]. Recently, Nemoto and Munro [4] proposed a scheme for realizing a nearly deterministic two-photon parity gate with weak XKNLs, in which the nonlinearity effect is 'amplified' by an intense coherent state bus. Thereafter, similar schemes for implementing photonic two-qubit parity and 
CNOT gates were developed [32-36]. All these schemes involve a building block of successive cross-Kerr interactions between an intense coherent state probe beam-that acts as a quantum information bus-and a pair of singlephoton qubit beams. To make these schemes work in the regime of $\theta \sim 10^{-2}$, giant intensities of coherent states or rounds of operations (each round involves two cross-Kerr interactions) are required. These requirements, however, are very difficult to achieve in the experiment, or even go beyond the reasonability. In addition, it is still an experimental challenge to achieve such a value of XKPS. This idea has also been widely employed in the research on the Bell-state measurement, generation of entangled states and coherent state superpositions, and so on (see, e.g., [37-44]]).

In this paper, we propose a scheme for realizing near deterministically a photonic two-qubit parity gate using a tiny XKNL. The involved XKNL can be several orders of magnitude weaker than the aforementioned schemes. The scheme employs an optical device based on a high quality ring cavity coupled to an external traveling wave (signal mode) through a cross-Kerr medium [42]. The cavity mode is fed by a coherent state and serves as a probe, and the detection outcomes at the cavity output ports reveal the parity (even parity or odd parity) of the two qubits without destroying the photons.

The paper is organized as follows. In Sec. II, we introduce the idea of performing a two-qubit parity gate with a tiny XKNL. In Sec. III, we discuss the effects of some nonideal cases on the parity gate: Sec. III A focuses on the imperfection of the detectors, and Sec. III B on the photon losses of the coherent state bus. Finally, concluding remarks are given in Sec. IV.

\section{TWO-QUBIT PARITY GATE}

The schematic setup of realizing the two-qubit parity gate is depicted in Fig. 1. For simplicity, we suppose that the two beam splitters (BSs) have the same transmissivity $\tau$ and their absorptions are negligible. The cavity is fed by a coherent state $|\alpha\rangle$ in the input mode $i_{1}$, whereas the input mode $i_{2}$ is left unexcited. After passing through the polarizing beam splitter (PBS) $P B S_{1}$, each of the two polarized photons will be in one of the path modes $s_{1}$ and $s_{2}$, which depends on their polarization states. The mode $s_{1}$ (signal mode) is coupled to the cavity mode through the cross-Kerr medium. The two output ports of the cavity are monitored, respectively, by the photon-number resolving (PNR) detectors $D_{1}$ and $D_{2}$ (when a certain condition is satisfied, $D_{2}$ can be omitted as shown later). $P B S_{2}$ serves as separating the two photons into different spatial modes.

Before describing the performance of the parity gate in more detail, we first analyze the dependence of the states of the modes $o_{1}$ and $o_{2}$ on the state of the mode $s_{1}$. The input-output relations for the cavity are given by [42, 43]

$$
\begin{aligned}
& a_{o_{1}}^{+}=\kappa_{n} a_{i_{1}}^{+}+\exp [i(1-n) \theta] \sigma_{n} a_{i_{2}}^{+}, \\
& a_{o_{2}}^{+}=\sigma_{n} a_{i_{1}}^{+}+\kappa_{n} a_{i_{2}}^{+},
\end{aligned}
$$

where the cavity transmissivity $\sigma_{n}$ and reflectivity $\kappa_{n}$ are dependent on the photon number $n$ in the signal mode $s_{1}$,

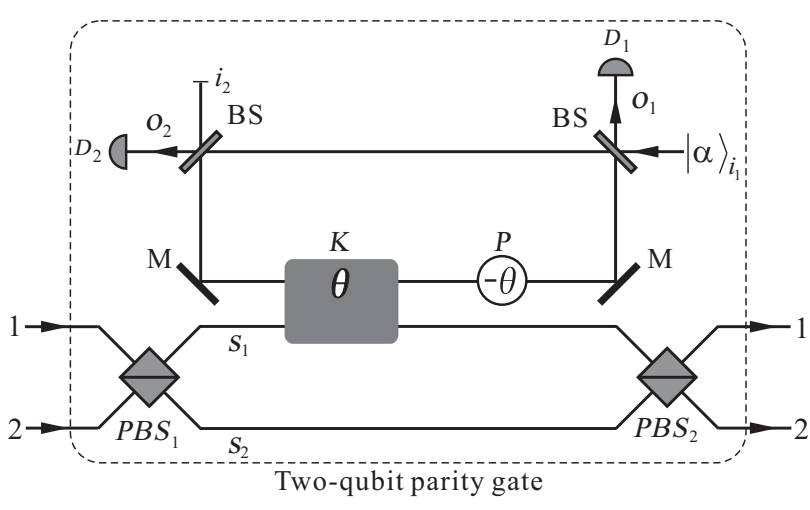

FIG. 1: Schematic setup for the two-qubit parity gate. $P B S_{1}$ and $P B S_{2}$ are two polarizing beam splitters, which transmit the horizontal polarization component and reflect the vertical component. Two BSs with low transmissivity $\tau$ and two mirrors (M) constitute a ring cavity which is fed by a coherent state in the mode $i_{1}$. $K$ is a cross-Kerr medium by which every photon in the mode $s_{1}$ induces a phase shift $\theta$ on the coherent state. $P$ is a static phase shifter that puts a phase shift $-\theta$ on the coherent state. The cavity transmissivity and reflectivity are dependent on the phase shift on the coherent state. $D_{1}$ and $D_{2}$ are two photon-number resolving detectors, and their detection outcomes reveal the parity (even parity or odd parity) of the two qubits without destroying the photons. Note that a simple local rotation (which depends on the outcomes of $D_{1}$ and $D_{2}$ ) on qubit 1 or 2 via a feedforward process is not shown in the sketch.

both of which read

$$
\begin{aligned}
& \kappa_{n}=\frac{\sqrt{1-\tau}\{\exp [i(1-n) \theta]-1\}}{1-(1-\tau) \exp [i(1-n) \theta]} \\
& \sigma_{n}=\frac{\tau}{1-(1-\tau) \exp [i(1-n) \theta]}, \\
& \left|\sigma_{n}\right|^{2}=\left[1+4 \frac{1-\tau}{\tau^{2}} \sin ^{2} \frac{(1-n) \theta}{2}\right]^{-1}, \\
& \left|\kappa_{n}\right|^{2}=1-\left|\sigma_{n}\right|^{2}
\end{aligned}
$$

Suppose that the mode $s_{1}$ is initially in an entangled state with the mode $s_{2}$,

$$
|\psi\rangle_{s_{1} s_{2}}=\sum_{n, m} C_{n m}|n\rangle_{s_{1}}|m\rangle_{s_{2}}
$$

where $|n\rangle_{s_{1}}$ and $|m\rangle_{s_{2}}$ denote the photon-number or Fock states of the modes $s_{1}$ and $s_{2}$, respectively. Then the input state of the total system is

$$
|\psi\rangle_{s_{1} s_{2} i_{1} i_{2}}=\sum_{n, m} C_{n m}|n\rangle_{s_{1}}|m\rangle_{s_{2}}|\alpha\rangle_{i_{1}}|0\rangle_{i_{2}}
$$

According to Eq. (1), we can obtain the output state of the total system,

$$
|\psi\rangle_{s_{1} s_{2} o_{1} o_{2}}=\sum_{n, m} C_{n m}|n\rangle_{s_{1}}|m\rangle_{s_{2}}\left|\kappa_{n} \alpha\right\rangle_{o_{1}}\left|\sigma_{n} \alpha\right\rangle_{o_{2}} .
$$

Now we consider the two-qubit parity gate. Assume that two polarization qubits are initially in the state

$$
\begin{aligned}
\left|\phi^{i n}\right\rangle_{12}= & x_{0}|H\rangle_{1}|H\rangle_{2}+x_{1}|H\rangle_{1}|V\rangle_{2} \\
& +x_{2}|V\rangle_{1}|H\rangle_{2}+x_{3}|V\rangle_{1}|V\rangle_{2},
\end{aligned}
$$


where $H$ and $V$ denote the horizontal and vertical polarizations, respectively. This state may be separable or entangled depending on whether they have interacted previously. Because the PBSs transmit the horizontal polarization component and reflect the vertical component, after passing through $P B S_{1}$ (see Fig. 1) the incident state becomes

$$
\begin{aligned}
|\phi\rangle_{s_{1} s_{2}}= & x_{0}|H\rangle_{s_{1}}|H\rangle_{s_{2}}+x_{3}|V\rangle_{s_{1}}|V\rangle_{s_{2}} \\
& +x_{1}|0\rangle_{s_{1}}|H V\rangle_{s_{2}}+x_{2}|V H\rangle_{s_{1}}|0\rangle_{s_{2}} \\
= & x_{0}|1\rangle_{s_{1}}|1\rangle_{s_{2}}+x_{3}|1\rangle_{s_{1}}|1\rangle_{s_{2}} \\
& +x_{1}|0\rangle_{s_{1}}|2\rangle_{s_{2}}+x_{2}|2\rangle_{s_{1}}|0\rangle_{s_{2}}
\end{aligned}
$$

It can be seen that there is only one photon in each path mode (called balanced) for the initial state $|H\rangle_{1}|H\rangle_{2}$ or $|V\rangle_{1}|V\rangle_{2}$, while there are two photons in one path mode and none in the other (called bunched) for the initial state $|H\rangle_{1}|V\rangle_{2}$ or $|V\rangle_{1}|H\rangle_{2}$. The mode $s_{1}$ is then coupled to the cavity mode through a cross-Kerr medium, as shown in Fig. 1. The subsequent $P B S_{2}$ is used to separate the two photons into different spatial modes. According to Eq. (5), the whole system will be finally in the state

$$
\begin{aligned}
|\phi\rangle_{12 o_{1} o_{2}}= & \left(x_{0}|H\rangle_{1}|H\rangle_{2}+x_{3}|V\rangle_{1}|V\rangle_{2}\right)\left|\kappa_{1} \alpha\right\rangle_{o_{1}}\left|\sigma_{1} \alpha\right\rangle_{o_{2}} \\
& +x_{1}|H\rangle_{1}|V\rangle_{2}\left|\kappa_{0} \alpha\right\rangle_{o_{1}}\left|\sigma_{0} \alpha\right\rangle_{o_{2}} \\
& +x_{2}|V\rangle_{1}|H\rangle_{2}\left|\kappa_{2} \alpha\right\rangle_{o_{1}}\left|\sigma_{2} \alpha\right\rangle_{o_{2}} .
\end{aligned}
$$

We observe immediately from Eq. (2) that $\kappa_{1}=0, \sigma_{1}=1$, $\kappa_{0}=\kappa_{2}^{*}=\kappa$, and $\sigma_{0}=\sigma_{2}^{*}=\sigma$. Note that $\kappa_{1}=0$ and $\sigma_{1}=1$ means the cavity being at resonance and having unit transmissivity [43]. Thus the state of Eq. (8) reduces to

$$
\begin{aligned}
|\phi\rangle_{12 o_{1} o_{2}}= & \left(x_{0}|H\rangle_{1}|H\rangle_{2}+x_{3}|V\rangle_{1}|V\rangle_{2}\right)|0\rangle_{o_{1}}|\alpha\rangle_{o_{2}} \\
& +x_{1}|H\rangle_{1}|V\rangle_{2}|\kappa \alpha\rangle_{o_{1}}|\sigma \alpha\rangle_{o_{2}} \\
& +x_{2}|V\rangle_{1}|H\rangle_{2}\left|\kappa^{*} \alpha\right\rangle_{o_{1}}\left|\sigma^{*} \alpha\right\rangle_{o_{2}}
\end{aligned}
$$

For implementing the two-qubit parity gate, that is, obtaining the even parity state (non-normalized)

$$
\left|\phi^{e v e n}\right\rangle_{12}=x_{0}|H\rangle_{1}|H\rangle_{2}+x_{3}|V\rangle_{1}|V\rangle_{2}
$$

(two photons have the same polarization and are correlated with each other) or the odd parity state (non-normalized)

$$
\left|\phi^{\text {odd }}\right\rangle_{12}=x_{1}|H\rangle_{1}|V\rangle_{2}+x_{2}|V\rangle_{1}|H\rangle_{2}
$$

(two photons have different polarizations and are anticorrelated with each other), we need to distinguish the probe state $|0\rangle_{o_{1}}|\alpha\rangle_{O_{2}}$ from $|\kappa \alpha\rangle_{o_{1}}|\sigma \alpha\rangle_{O_{2}}$ and $\left|\kappa^{*} \alpha\right\rangle_{o_{1}}\left|\sigma^{*} \alpha\right\rangle_{o_{2}}$, but not (even in principle) distinguish $|\kappa \alpha\rangle_{o_{1}}|\sigma \alpha\rangle_{o_{2}}$ from $\left|\kappa^{*} \alpha\right\rangle_{o_{1}}\left|\sigma^{*} \alpha\right\rangle_{o_{2}}$. It will be shown that this task can be accomplished by detecting the photon numbers of the modes $o_{1}$ and $o_{2}$ with the PNR detectors $D_{1}$ and $D_{2}$, respectively.

The indistinguishability between $|\kappa \alpha\rangle_{o_{1}}$ and $\left|\kappa^{*} \alpha\right\rangle_{o_{1}}$ with photon-number detection is evident. The same is true of the indistinguishability between $|\sigma \alpha\rangle_{o_{2}}$ and $\left|\sigma^{*} \alpha\right\rangle_{o_{2}}$. For distinguishing $|0\rangle_{o_{1}}|\alpha\rangle_{o_{2}}$ from $|\kappa \alpha\rangle_{o_{1}}|\sigma \alpha\rangle_{o_{2}}$ and $\left|\kappa^{*} \alpha\right\rangle_{o_{1}}\left|\sigma^{*} \alpha\right\rangle_{o_{2}}$, we in fact only need to distinguish $|0\rangle_{o_{1}}$ from $|\kappa \alpha\rangle_{o_{1}}$ or $\left|\kappa^{*} \alpha\right\rangle_{o_{1}}$. It will be shown later that the photon-number detection on the mode $o_{2}$ is just for removing the relative phase shift between the two components of the odd parity state. The overlap between $|\kappa \alpha\rangle_{o_{1}}$ and $|0\rangle_{o_{1}}$ is very small and negligible if the amplitude $\kappa \alpha$ is large enough, and they can be well distinguished from each other. The lower limit of $|\kappa \alpha|$ depends on the quantum efficiency of the detector $D_{1}$ and the allowable error rate. For clarity, we first assume both $D_{1}$ and $D_{2}$ have unity quantum efficiency, and the imperfect case will be discussed later. Then a measurement on the mode $o_{2}$ projects the two qubits and the mode $o_{2}$ in the state (non-normalized)

$$
\left|\phi^{e}\right\rangle \approx\left(x_{0}|H\rangle_{1}|H\rangle_{2}+x_{3}|V\rangle_{1}|V\rangle_{2}\right)|\alpha\rangle_{o_{2}}
$$

for the photon number $n_{o_{1}}=0$, or (non-normalized)

$$
\begin{aligned}
\left|\phi^{o}\right\rangle= & x_{1} e^{i n_{o_{1}} \arg (\kappa)}|H\rangle_{1}|V\rangle_{2}|\sigma \alpha\rangle_{o_{2}} \\
& +x_{2} e^{-i n_{o_{1}} \arg (\kappa)}|V\rangle_{1}|H\rangle_{2}\left|\sigma^{*} \alpha\right\rangle_{o_{2}} \\
= & \sum_{n_{o_{2}}=0}^{\infty} f_{n_{o_{2}}}\left\{x_{1} e^{i \varphi\left(n_{o_{1}}, n_{o_{2}}\right)}|H\rangle_{1}|V\rangle_{2}\right. \\
& \left.+x_{2} e^{-i \varphi\left(n_{o_{1}}, n_{o_{2}}\right)}|V\rangle_{1}|H\rangle_{2}\right\}\left|n_{o_{2}}\right\rangle
\end{aligned}
$$

for $n_{o_{1}}>0$, where $f_{n_{o_{2}}}=e^{-|\sigma \alpha|^{2} / 2}(|\sigma| \alpha)^{n_{o_{2}}} / \sqrt{n_{o_{2}} !}$, $\varphi\left(n_{o_{1}}, n_{o_{2}}\right)=n_{o_{1}} \operatorname{Arg}(\kappa)+n_{o_{2}} \operatorname{Arg}(\sigma)$, and the identities $\arg \left(\kappa^{*}\right) \equiv-\operatorname{Arg}(\kappa)$ and $\arg \left(\sigma^{*}\right) \equiv-\operatorname{Arg}(\sigma)$ have been utilized. We have used the approximate equality $(\approx)$ in Eq. $(12)$ as there is a small but finite probability that the state (13) can also occur for $n_{o_{1}}=0$. If $\left|x_{0}\right|=\left|x_{1}\right|=\left|x_{2}\right|=\left|x_{3}\right|=1 / 2$ (without loss of generality, we shall take the same value in the following context), the probability of this error occurring is given by

$$
P_{\text {err }}=\frac{1}{2}|\langle 0 \mid \kappa \alpha\rangle|^{2}=\frac{1}{2} \exp \left(-|\kappa \alpha|^{2}\right),
$$

which is less than $10^{-4}$ when $|\kappa \alpha|>3$. Thus, $|\kappa \alpha\rangle_{o_{1}}$ and $|0\rangle_{o_{1}}$ can be discriminated near deterministically. Experimental implementations of discriminating between a coherent state and a vacuum state using the photon-number measurement have been recently reported [44, 45].

It can be seen that for the measurement outcome $n_{o_{1}}=0$ the mode $o_{2}$ is disentangled and the two qubits are directly projected in the even parity state of Eq. (10). However, for $n_{o_{1}}>0$ the two qubits are still entangled with the mode $o_{2}$. To disentangle the mode $o_{2}$, one needs to perform another photon-number measurement on $o_{2}$ and obtain its photon number $n_{O_{2}}$. Then one obtains an odd parity state with the two components picking up an unwanted relative phase shift $2 \varphi\left(n_{o_{1}}, n_{o_{2}}\right)$. The phase shift $2 \varphi\left(n_{o_{1}}, n_{o_{2}}\right)$ can be eliminated via a classical feedforward operation (In many computational circuits the phase-shift removing operations can be delayed and performed at the final measurement stage for the qubits). After all the operations discussed above, we can conclude that a two-qubit parity gate is accomplished with near one probability.

As shown above, detecting the mode $o_{1}$ is sufficient for discriminating between the odd and even parities of the two qubits, and detecting $\mathrm{O}_{2}$ is just for removing the relative phase shift of the two odd parity components $|H\rangle_{1}|V\rangle_{2}$ and $|V\rangle_{1}|H\rangle_{2}$. When $|\sigma \alpha|$ is very small, the mean photon number $\left(\bar{n}_{O_{2}}\right)$ of the states $|\sigma \alpha\rangle$ and $\left|\sigma^{*} \alpha\right\rangle$ is close to zero. Then one may omit the detection of $o_{2}$. This, however, will yield a small error probability given by $1-|\langle 0 \mid \sigma \alpha\rangle|^{2}=$ $1-\exp \left(-\bar{n}_{O_{2}}^{2}\right)$ which is less than $10^{-3}$ when $\bar{n}_{O_{2}}<0.001$. 
In what follows, we analyze how large XKPS $(\theta)$ is required for realizing the aforementioned parity gate. For a certain value of $P_{e r r}$, the value of $\theta$ evidently depends on the values of $|\alpha|$ and $\tau$. What we are interested in is the weak nonlinearity regime (i.e., $\theta \ll 1$ ). In addition, we assume the transmissivity $(\tau)$ of the BSs is also very small. Then, $|\kappa|\left(=\left|\kappa_{0}\right|=\left|\kappa_{2}\right|\right)$ and $|\sigma|\left(=\left|\sigma_{0}\right|=\left|\sigma_{2}\right|\right)$ can be approximated as

$$
\begin{aligned}
& |\kappa|^{2} \approx \frac{r^{2}}{r^{2}+1}, \\
& |\sigma|^{2} \approx \frac{1}{r^{2}+1},
\end{aligned}
$$

where $r=\theta / \tau$. According to Eqs. (14) and (15), the relationship of $r$ and $|\alpha|$ is given by

$$
|\alpha| \approx \frac{1}{r} \sqrt{\left(r^{2}+1\right) \ln \frac{1}{2 P_{e r r}}} .
$$

Evidently, $r$ decreases (i.e., $\theta$ decreases for a given $\tau$ ) as $|\alpha|$ increases for a certain value of $P_{e r r}$, and vice versa. When $P_{\text {err }}$ and $|\alpha|$ are given (i.e., $r$ is given), $\theta$ is in inverse proportion to $1 / \tau$. These results imply that our scheme can work in the regime of tiny XKNL.

We take $P_{\text {err }}=10^{-4}$ as an example. Then the dependence relation of $r$ and $|\alpha|$ is shown in Fig. 2. It can be seen that $|\alpha|$ slowly increases as $r$ rapidly decreases in the range $r \gtrsim 2$, while $|\alpha|$ rapidly increases as $r$ slowly decreases in the range $r \lesssim 0.5$. This indicates that a large decrease in $\theta$ only needs a small increase in $|\alpha|$ for the case $\theta \gtrsim 2 \tau$, while a small decrease in $\theta$ needs a large increase in $|\alpha|$ for the case $\theta \lesssim \tau / 2$. Thus there is a trade-off between $\theta$ and $|\alpha|$. In the following context, we focus on an example case, $\theta=\tau$. Then the lower $\tau$ is, the smaller regime of $\theta$ our scheme can work in. For example, when $\tau$ is of the order of $\left\{10^{-6}, 10^{-5}, 10^{-4}\right\}$ (these values are available under current technology [42, 46]), $\theta$ is correspondingly of the order of $\left\{10^{-6}, 10^{-5}, 10^{-4}\right\}$. It seems that the previous schemes can also work in the regime of these orders of magnitude of XKPS by increasing the amplitude of the probe coherent state or the number of round. However, the intensity of the coherent state or the number of round would go beyond the accessible or even reasonable values. For example, to make the parity gate operate properly even for $\theta \sim 10^{-2}$ (assuming the error probability is also of the order of $10^{-4}$ ), the amplitude of the probe coherent state should be $\sim 10^{4}$ in Refs. [4, 32, 33] (note that a much more intense ancillary coherent state beam is also required for accomplishing the homodyne measurement on the probe coherent state beam [47]), and the number of round (each round involves two cross-Kerr interactions) should also be $\sim 10^{4}$ in Ref. [34]. Although the amplitude of the probe coherent state can be reduced to a certain extent by using photon-number measurement than homodyne measurement [34-36], inaccessibly or even unreasonably intense ancillary coherent state would be required for accomplishing an appropriate displacement on the probe coherent state. Thus we conclude that our scheme can work in the regime of a several orders of magnitude weaker XKNL than those schemes mentioned above.

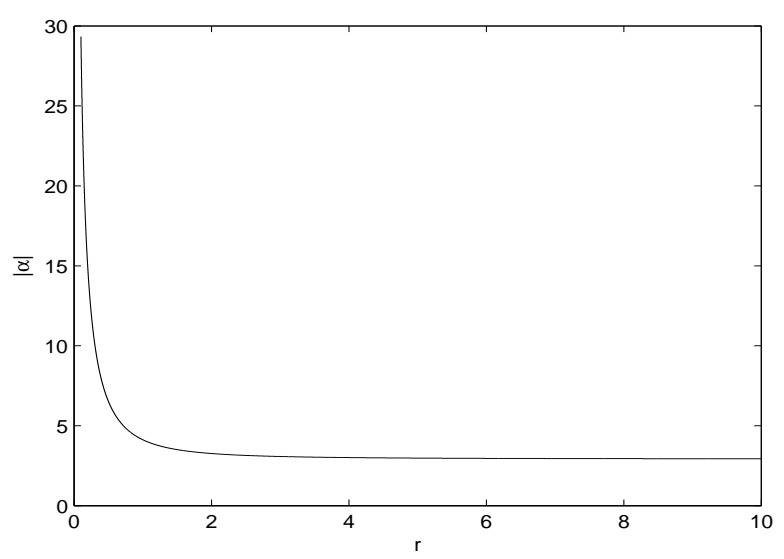

FIG. 2: The dependence relation of $|\alpha|$ and $r$, with the error probability $P_{\text {err }}=10^{-4}$.

\section{EFFECTS OF NONIDEAL CASES ON THE TWO-QUBIT PARITY GATE}

\section{A. Imperfections of detectors}

We now consider that the two detectors $D_{1}$ and $D_{2}$ have nonunit quantum efficiencies $\eta_{1}$ and $\eta_{2}$, respectively. Then the error probability (14) is replaced by

$$
\begin{aligned}
P_{e r r}^{\left(\eta_{1}\right)} & =\frac{1}{2} \operatorname{Tr}\left[|\kappa \alpha\rangle\left\langle\kappa \alpha\left|\sum_{n=0}^{\infty}\left(1-\eta_{1}\right)^{n}\right| n\right\rangle\langle n|\right] \\
& =\frac{1}{2} \exp \left[-\eta_{1}|\kappa \alpha|^{2}\right] .
\end{aligned}
$$

Evidently, the imperfection of $D_{1}$ can be well compensated by slightly increasing $|\alpha|$. For the same $\kappa, P_{e r r}^{\left(\eta_{1}\right)}\left(\alpha^{\prime}\right)=$ $P_{\text {err }}(\alpha)$ with $\alpha^{\prime}=\alpha / \sqrt{\eta_{1}}$, and when, for example, $\eta_{1}=$ $0.9,\left|\alpha^{\prime}\right|$ is about 4.22 for $|\alpha|=4$ and about 30.57 for $|\alpha|=$ 29.

For eliminating the relative phase shift of the two odd parity components, one needs to know the correct photon numbers of the modes $o_{1}$ and $o_{2}$, as shown before. The imperfections of $D_{1}$ and $D_{2}$ will cause an error probability given by

$$
\begin{aligned}
P_{\text {err }}^{\prime\left(\eta_{1}+\eta_{2}\right)} & =1-\sum_{n=1}^{\infty}|\langle n \mid \kappa \alpha\rangle|^{2} \eta_{1}^{n} \sum_{m=1}^{\infty}|\langle m \mid \sigma \alpha\rangle|^{2} \eta_{2}^{m} \\
& =1-\exp \left[\left(\eta_{1}|\kappa|^{2}+\eta_{2}|\sigma|^{2}-1\right)|\alpha|^{2}\right] .(18)
\end{aligned}
$$

For simplicity, we suppose $\eta_{1}=\eta_{2}=\eta$. Then the above equation reduces to

$$
P_{e r r}^{\prime(\eta)}=1-\exp \left[(\eta-1)|\alpha|^{2}\right]
$$

which is independent of the XKPS $\theta . P_{e r r}^{\prime(\eta)}$ is small if and only if $(1-\eta)|\alpha|^{2}$ is small. When $(1-\eta)|\alpha|^{2}<0.05, P_{e r r}^{\prime(\eta)}$ is less than 0.05. In addition, for a given value of $\eta$, the larger $|\alpha|$ is, the larger $P_{e r r}^{\prime(\eta)}$ becomes. Thus, for making $P_{e r r}^{\prime(\eta)}$ be very small, the quantum efficiency is required to be very high when $|\alpha|$ is not very small. Fortunately, recent reports [48-50] indicated that $\eta$ could be close to unity with the developing techniques. We notice that potential dark 
counts of the detectors could also cause errors. However, recent experiments [48-50] demonstrated that near-unityefficiency PNR detectors with negligible dark-count rates could be produced. Note that other schemes [4, 32-36] may be more fragile to the imperfection of the detectors, because they involve photon-number measurements on very intense coherent states as mentioned before.

It is worth stressing that $P_{e r r}^{\prime\left(\eta_{1}+\eta_{2}\right)}$ is not the error probability of distinguishing between the odd and even parities of the two qubits but the error probability of removing the relative phase shift of the two odd parity components. The error probability $\left(P_{e r r}^{\left(\eta_{1}\right)}\right)$ of distinguishing the odd parity components $|H V\rangle$ and $|V H\rangle$ from the even parity components $|H H\rangle$ and $|V V\rangle$ could be close to zero even when the detection efficiency is low (in the following context, we shall use the abbreviation $|H\rangle|V\rangle=|H V\rangle$ ). Therefore, even with low-efficiency detectors, our scheme could be used to implement the Bell-state measurement with near unity probability [32, 35, 51] and generate cluster states with a certain probability [32, 52] as well as serve all other quantum tasks that involve two-photon polarization-parity detections.

It has been mentioned before that when $|\sigma \alpha|$ is very small (e.g., $r=100$ and $|\alpha|$ is not too large), the measurement on the mode $O_{2}$ may well be omitted. Then the error probability $P_{e r r}^{\prime\left(\eta_{1}+\eta_{2}\right)}$ vanishes.

\section{B. Photon losses in the bus}

In this section, we discuss the decoherence effect due to photon absorption in the cross-Kerr medium. Photon losses may occur in both the bus and the qubit modes. However, photon losses in the coherent state field is more easier to occur. Therefore, photon losses of the coherent state field should be the main source of decoherence in the twoqubit output state when the interaction time is very short [20, 35, 39]. In what follows, we shall consider the photon losses in the bus. Such photon losses can be modeled via a beam splitter of transmissivity $\lambda$ which discards a portion of the coherent state beam [35, 53]. It is assumed that $\lambda$ does not vary with time and can be measured in advance through suitable test experiments [20, 35], so its value is known. Then Fig. 1 can be altered phenomenally to Fig. 3, where the beam splitter $B S^{\prime}$ (with transmissivity $\lambda$ ) is to model the photon losses in the cavity field. Following the method of the authors of [42, 43], we obtain the input-output relations for the cavity,

$$
\begin{aligned}
& a_{o_{1}}^{+}=A_{n} a_{i_{1}}^{+}+\frac{\tau \sqrt{1-\lambda} e^{i(1-n) \theta}}{\Gamma} a_{i_{2}}^{+}+\frac{\sqrt{\tau \lambda}}{\Gamma} a_{i_{3}}^{+}, \\
& a_{o_{2}}^{+}=B_{n} a_{i_{1}}^{+}+A_{n} a_{i_{2}}^{+}+\frac{\sqrt{\lambda \tau(1-\tau)}}{\Gamma} a_{i_{3}}^{+}, \\
& a_{o_{3}}^{+}=C_{n} a_{i_{1}}^{+}+\frac{C_{n}}{\sqrt{1-\tau}} a_{i_{2}}^{+}+\frac{\rho e^{i(1-n) \theta}-\sqrt{1-\lambda}}{\Gamma} a_{i_{3}}^{+},
\end{aligned}
$$

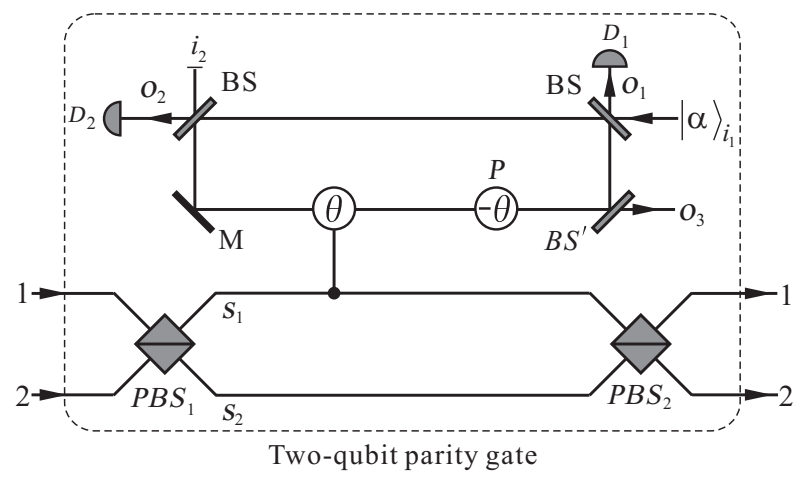

FIG. 3: Diagrammatic sketch of the two-qubit parity gate. Each photon in the mode $s_{1}$ induces a phase shift $\theta$ on the coherent state through the cross-Kerr medium. The additional beam splitter $B S^{\prime}$ (with transmissivity $\lambda$ ) is used to model the photon losses in the coherent state bus.

where

$$
\begin{aligned}
& \Gamma=1-(1-\tau) \sqrt{1-\lambda} e^{i(1-n) \theta}, \\
& A_{n}=\frac{\sqrt{1-\tau}\left[\sqrt{1-\lambda} e^{i(1-n) \theta}-1\right]}{\Gamma}, \\
& B_{n}=\frac{\tau}{\Gamma}, \\
& C_{n}=\frac{\sqrt{\lambda \tau(1-\tau)} e^{i(1-n) \theta}}{\Gamma},
\end{aligned}
$$

and $n(=0,1,2)$ is the photon number in the mode $s_{1}$. The mode $o_{3}$ that denotes the photon losses in the cavity field has to be traced out [35, 53]. Then, the output state of Eq. (9) is replaced by

$$
\begin{aligned}
\rho_{12 o_{1} o_{2}}= & \left|\phi^{\text {even }}\right\rangle_{12}\left\langle\phi^{\text {even }}|\otimes| A_{1} \alpha\right\rangle_{o_{1}}\left\langle A_{1} \alpha|\otimes| B_{1} \alpha\right\rangle_{o_{2}}\left\langle B_{1} \alpha\right| \\
& +\left|x_{1}\right|^{2}|H V\rangle_{12}\left\langle H V|\otimes| A_{0} \alpha\right\rangle_{o_{1}}\left\langle A_{0} \alpha|\otimes| B_{0} \alpha\right\rangle_{o_{2}}\left\langle B_{0} \alpha\right| \\
& +\left|x_{2}\right|^{2}|V H\rangle_{12}\left\langle V H|\otimes| A_{0}^{*} \alpha\right\rangle_{o_{1}}\left\langle A_{0}^{*} \alpha|\otimes| B_{0}^{*} \alpha\right\rangle_{o_{2}}\left\langle B_{0}^{*} \alpha\right| \\
& +x_{1}^{*} y_{1}\left|\phi^{\text {even }}\right\rangle_{12}\left\langle H V|\otimes| A_{1} \alpha\right\rangle_{o_{1}}\left\langle A_{0} \alpha|\otimes| B_{1} \alpha\right\rangle_{o_{2}}\left\langle B_{0} \alpha\right| \\
& +x_{1} y_{1}|H V\rangle_{12}\left\langle\phi^{\text {even }}|\otimes| A_{0} \alpha\right\rangle_{o_{1}}\left\langle A_{1} \alpha|\otimes| B_{0} \alpha\right\rangle_{o_{2}}\left\langle B_{1} \alpha\right| \\
& +x_{2}^{*} y_{2}\left|\phi^{\text {even }}\right\rangle_{12}\left\langle V H|\otimes| A_{1} \alpha\right\rangle_{o_{1}}\left\langle A_{0}^{*} \alpha|\otimes| B_{1} \alpha\right\rangle_{o_{2}}\left\langle B_{0}^{*} \alpha\right| \\
& +x_{2} y_{2}|V H\rangle_{12}\left\langle\phi^{\text {even }}|\otimes| A_{0}^{*} \alpha\right\rangle_{o_{1}}\left\langle A_{1} \alpha|\otimes| B_{0}^{*} \alpha\right\rangle_{o_{2}}\left\langle B_{1} \alpha\right| \\
& +x_{1} x_{2}^{*} y_{3}|H V\rangle_{12}\left\langle V H|\otimes| A_{0} \alpha\right\rangle_{o_{1}}\left\langle A_{0}^{*} \alpha|\otimes| B_{0} \alpha\right\rangle_{o_{2}}\left\langle B_{0}^{*} \alpha\right| \\
& +x_{1}^{*} x_{2} y_{3}|V H\rangle_{12}\left\langle H V|\otimes| A_{0}^{*} \alpha\right\rangle_{o_{1}}\left\langle A_{0} \alpha|\otimes| B_{0}^{*} \alpha\right\rangle_{o_{2}}\left\langle B_{0} \alpha\right|,
\end{aligned}
$$


where

$$
y_{1}=\left|\left\langle C_{0} \alpha \mid C_{1} \alpha\right\rangle\right|^{2}, \quad y_{2}=\left|\left\langle C_{0}^{*} \alpha \mid C_{1} \alpha\right\rangle\right|^{2}, \quad y_{3}=\left|\left\langle C_{0}^{*} \alpha \mid C_{0} \alpha\right\rangle\right|^{2},
$$

and the relations $A_{2}=A_{0}^{*}$ and $B_{2}=B_{0}^{*}$ have been utilized. In this case, the probe mode $o_{1}$ must be displaced by an amount $D\left(-A_{1} \alpha\right)=\exp \left(A_{1} \alpha^{*} a_{o_{1}}-A_{1} \alpha a_{o_{1}}^{+}\right)\left(A_{1}^{*}=A_{1}\right)$ prior to the measurement. Then the state of Eq. (22) evolves to

$$
\begin{aligned}
& \rho_{12 o_{1} o_{2}}=\left|\phi^{e v e n}\right\rangle_{12}\left\langle\phi^{e v e n}|\otimes| 0\right\rangle_{o_{1}}\left\langle 0|\otimes| B_{1} \alpha\right\rangle_{o_{2}}\left\langle B_{1} \alpha\right| \\
& +\left|x_{1}\right|^{2}|H V\rangle_{12}\left\langle H V|\otimes|\left(A_{0}-A_{1}\right) \alpha\right\rangle_{o_{1}}\left\langle\left(A_{0}-A_{1}\right) \alpha|\otimes| B_{0} \alpha\right\rangle_{o_{2}}\left\langle B_{0} \alpha\right| \\
& +\left|x_{2}\right|^{2}|V H\rangle_{12}\left\langle V H|\otimes|\left(A_{0}-A_{1}\right)^{*} \alpha\right\rangle_{o_{1}}\left\langle\left(A_{0}-A_{1}\right)^{*} \alpha|\otimes| B_{0}^{*} \alpha\right\rangle_{o_{2}}\left\langle B_{0}^{*} \alpha\right| \\
& +x_{1}^{*} y_{1} e^{i|\alpha|^{2} A_{1} \operatorname{Im} A_{0}}\left|\phi^{e v e n}\right\rangle_{12}\langle H V|\otimes| 0\rangle_{o_{1}}\left\langle\left(A_{0}-A_{1}\right) \alpha|\otimes| B_{1} \alpha\right\rangle_{o_{2}}\left\langle B_{0} \alpha\right| \\
& +x_{1} y_{1} e^{-i|\alpha|^{2} A_{1} \operatorname{Im} A_{0}}|H V\rangle_{12}\left\langle\phi^{e v e n}|\otimes|\left(A_{0}-A_{1}\right) \alpha\right\rangle_{o_{1}}\left\langle 0|\otimes| B_{0} \alpha\right\rangle_{o_{2}}\left\langle B_{1} \alpha\right| \\
& +x_{2}^{*} y_{2} e^{i|\alpha|^{2} A_{1} \operatorname{Im} A_{0}^{*}}\left|\phi^{e v e n}\right\rangle_{12}\langle V H|\otimes| 0\rangle_{o_{1}}\left\langle\left(A_{0}-A_{1}\right)^{*} \alpha|\otimes| B_{1} \alpha\right\rangle_{o_{2}}\left\langle B_{0}^{*} \alpha\right| \\
& +x_{2} y_{2} e^{-i|\alpha|^{2} A_{1} \operatorname{Im} A_{0}^{*}}|V H\rangle_{12}\left\langle\phi^{\text {even }}|\otimes|\left(A_{0}-A_{1}\right)^{*} \alpha\right\rangle_{o_{1}}\left\langle 0|\otimes| B_{0}^{*} \alpha\right\rangle_{o_{2}}\left\langle B_{1} \alpha\right| \\
& +x_{1} x_{2}^{*} y_{3} e^{-i 2|\alpha|^{2} A_{1} \operatorname{Im} A_{0}}|H V\rangle_{12}\left\langle V H|\otimes|\left(A_{0}-A_{1}\right) \alpha\right\rangle_{o_{1}}\left\langle\left(A_{0}-A_{1}\right)^{*} \alpha|\otimes| B_{0} \alpha\right\rangle_{o_{2}}\left\langle B_{0}^{*} \alpha\right| \\
& +x_{1}^{*} x_{2} y_{3} e^{i 2|\alpha|^{2} A_{1} \operatorname{Im} A_{0}}|V H\rangle_{12}\left\langle H V|\otimes|\left(A_{0}-A_{1}\right)^{*} \alpha\right\rangle_{o_{2}}\left\langle\left(A_{0}-A_{1}\right) \alpha|\otimes| B_{0}^{*} \alpha\right\rangle_{o_{2}}\left\langle B_{0} \alpha\right| .
\end{aligned}
$$

Note that the amplitudes of $|H V\rangle_{12}$ and $|V H\rangle_{12}$ have picked up a phase shift due to the displacement. These phase shifts are unwanted but can be simply removed by static phase shifters (no feedforward is required). After the operations as mentioned above (performing photon-number measurements on the modes $o_{1}$ and $o_{2}$, and eliminating the unwanted phase shifts via a classical feedforward process), the two qubits end in the even parity state $\left|\phi^{\text {even }}\right\rangle_{12}$ for $n_{o_{1}}=0$ or an odd parity state

$$
\rho_{12}^{\text {odd }}=\left|x_{1}\right|^{2}|H V\rangle_{12}\left\langle\left. H V|+| x_{2}\right|^{2} \mid V H\right\rangle_{12}\left\langle V H\left|+x_{1} x_{2}^{*} y_{3}\right| H V\right\rangle_{12}\left\langle V H\left|+x_{1}^{*} x_{2} y_{3}\right| V H\right\rangle_{12}\langle H V|
$$

for $n_{o_{1}}>0$. We have assumed that the detectors are perfect. Obviously, the potentially obtained even parity state is exactly the target state $\left|\phi^{e v e n}\right\rangle_{12}$ as given in Eq. (10), while the possibly obtained odd parity state is a mixed state which is different from the desired state $\left|\phi^{\text {odd }}\right\rangle_{12}$ as shown in Eq. (11). This indicates that the photon losses in the bus only cause decoherence for the odd parity state of the two qubits.

Let $\left|x_{0}\right|=\left|x_{1}\right|=\left|x_{2}\right|=\left|x_{3}\right|=1 / 2$. Then the error probability of distinguishing the even parity components $|H H\rangle$ and $|V V\rangle$ from the odd parity components $|H V\rangle$ and $|V H\rangle$ is

$$
\begin{aligned}
P_{e} & =\frac{1}{2}\left|\left\langle 0 \mid\left(A_{0}-A_{1}\right) \alpha\right\rangle\right|^{2} \\
& =\frac{1}{2} \exp \left\{\frac{2 \tau^{2}(1-\tau)(1-\lambda)(\cos \theta-1)|\alpha|^{2}}{\left[1-2(1-\tau) \sqrt{1-\lambda} \cos \theta+(1-\tau)^{2}(1-\lambda)\right][1-(1-\tau) \sqrt{1-\lambda}]^{2}}\right\} .
\end{aligned}
$$

Figure 4 shows that $P_{e}$ only depends on the ratio of $\lambda$ (photon loss parameter) to $\theta$ for a given $|\alpha|$ and $\theta=\tau$, and it universally decreases with the increase of $|\alpha|$. In a word, $P_{e}$ is approximate to zero and negligible when $\lambda$ does not exceed a certain threshold value depending in a nontrivial way upon the values of $\theta$ and $|\alpha|$. The overlap between the potentially obtained odd parity state and the desired odd parity state $\left|\phi^{\text {odd }}\right\rangle_{12}$ is given by

$$
\begin{aligned}
F_{\text {odd }} & =\frac{{ }_{12}\left\langle\phi^{\text {odd }}\left|\rho_{12}^{\text {odd }}\right| \phi^{\text {odd }}\right\rangle_{12}}{\operatorname{tr}\left(\left|\phi^{\text {odd }}\right\rangle_{12}\left\langle\phi^{\text {odd }}\right|\right) \operatorname{tr}\left(\rho_{12}^{\text {odd }}\right)} \\
& =\frac{1}{2}+\frac{1}{2} \exp \left\{\frac{-4 \tau(1-\tau) \lambda|\alpha|^{2} \sin ^{2} \theta}{\left[1-2(1-\tau) \sqrt{1-\lambda} \cos \theta+(1-\tau)^{2}(1-\lambda)\right]^{2}}\right\} .
\end{aligned}
$$

Figure 5 shows the dependence of $F_{\text {odd }}$ on $\lambda / \theta$ for a given $|\alpha|$ and $\theta=\tau$. It can be observed from figures 4 and 5 that the conditions of both $P_{e}$ being close to zero and $F_{o d d}$ being very large cannot be simultaneously satisfied when $\lambda / \theta$ is not sufficiently small.

As shown above, even when $F_{\text {odd }}$ holds small values, the even and odd parity components could be near deterministically distinguished. Similar to the foregoing case, our scheme with photon losses in the bus could be also used to implement the Bell-state measurement with near unity probability [32, 35, 51] and generate cluster states with a certain probability [32, 52] as well as serve all other quantum tasks that involve two-photon polarization-parity detections. Finally, we should point out that photon absorption in the cross-Kerr interactions could be nearly eliminated under certain conditions by using EIT materials [54].

Note that other potential factors should be also considered for practical implementation of our scheme, such as 

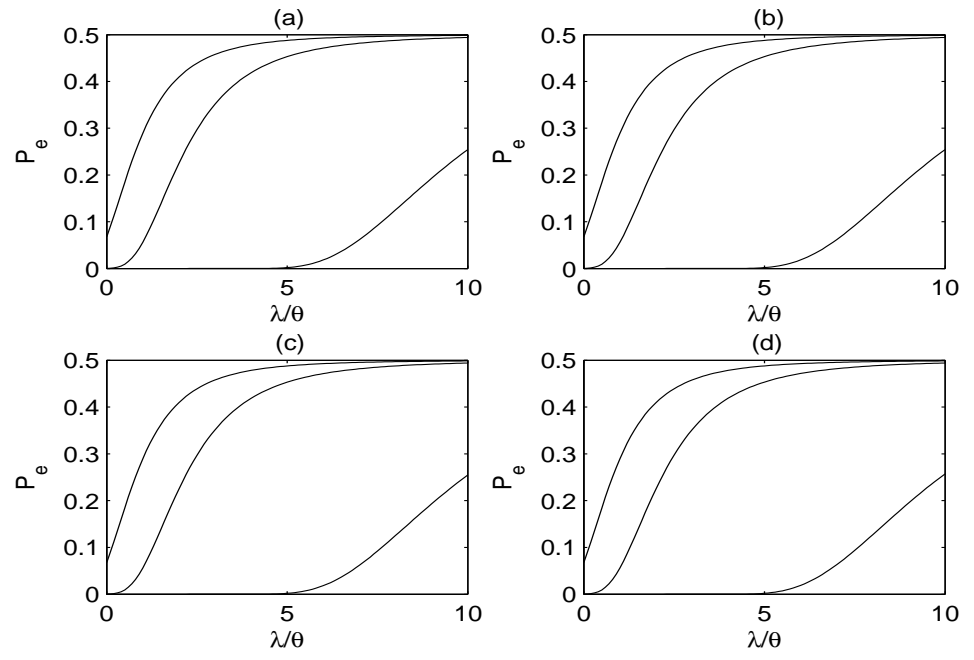

FIG. 4: The error probability $P_{e}$ against the ratio of $\lambda$ (photon loss parameter) to $\theta$. $\tau$ is equal to $\theta$. From top to bottom in each graph, the curves correspond to $|\alpha|=2,4$, and 30, respectively. (a) $\theta=10^{-6}$. (b) $\theta=10^{-5}$. (c) $\theta=10^{-4}$. (d) $\theta=10^{-3}$.
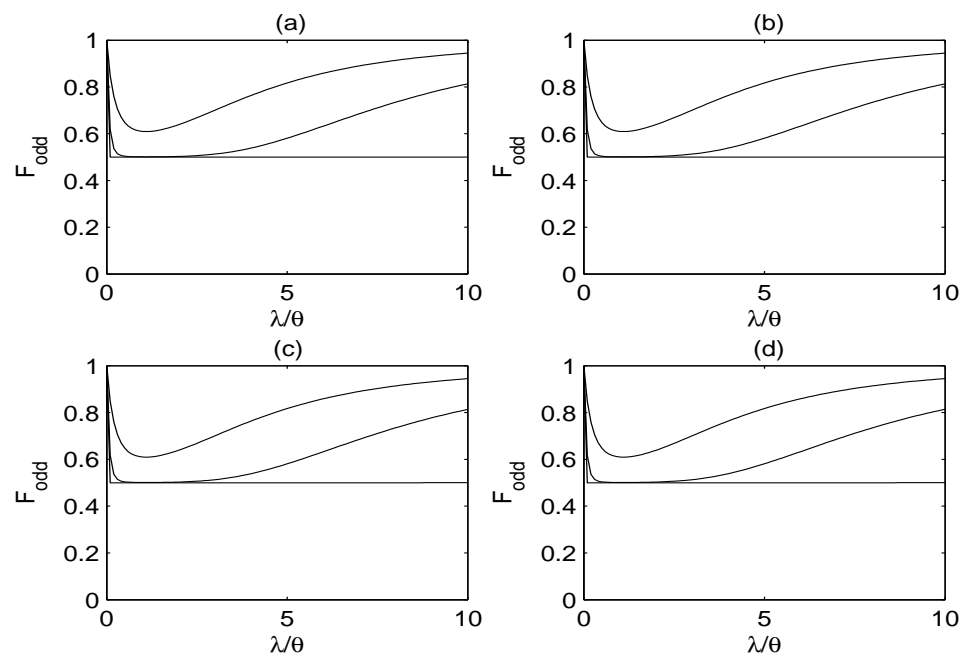

FIG. 5: The fidelity $F_{\text {odd }}$ against $\lambda / \theta . \tau$ is equal to $\theta$. From top to bottom in each graph, the curves correspond to $|\alpha|=2,4$, and 30 , respectively. (a) $\theta=10^{-6}$. (b) $\theta=10^{-5}$. (c) $\theta=10^{-4}$. (d) $\theta=10^{-3}$.

self-phase modulation and non-instantaneous response in the cross-Kerr medium. Fortunately, these effects may well be canceled through replacing fibers with EIT materials [21, 55]. In addition, the spectral effect can be also circumvented under certain conditions [55-57], and other resources of error can be dealt with using the standard techniques available for linear optical quantum computation [8].

\section{CONCLUDING REMARKS}

In conclusion, we have proposed a scheme for realizing a nearly deterministic two-photon polarization-parity gate using a tiny XKNL. Like previous XKNL-based schemes, our scheme only needs far fewer physical resources than linear optical schemes. The scheme employs an optical device based on a high quality ring cavity constructed by both two BSs and mirrors and fed by a coherent state. Such a device can substantially 'amplify' the nonlinearity effect, which makes it possible that our scheme work in the regime of a several orders of magnitude weaker XKNL than previous schemes. As a consequence, our scheme is more practical under current XKNL techniques. The presented two-qubit parity gate plus single-qubit rotations can constitute a universal gate set for economical and feasible all-optical quantum computation. Similarly, the two-qubit parity gate could serve quantum communication systems as it can be used to realize complete Bell-state measurements [32, 35, 51], multiphoton-entanglement generations [32, 52], optimal nonlocal multiphoton-entanglement concentrations [58], and so on. In addition, we showed that when the quantum efficiencies of the PNR detectors are less than one and there are photon losses in the bus, the even and odd parity states of the two polarization qubits can be near deterministically distinguished. These findings indicate that even in the nonideal cases, our scheme could efficiently serve the quantum tasks that involve two-photon polarization-parity detections. 


\section{Acknowledgments}

This work was supported by the National Natural Science Foundation of China (Grants No. 11004050 and 11075050), the Program for Changjiang Scholars and Innovative Research Team in University (Grant No. IRT0964), the Key Project of Chinese Ministry of Education (Grant No. 211119), the Scientific Research Fund of Hunan Provincial Education Department (Grants No. 09A013 and 10B013), and the construct program of the key discipline in Hunan province.
[1] R. Ionicioiu, Phys. Rev. A 75, 032339 (2007); R. Ionicioiu, A. E. Popescu, W. J. Munro, and T. P. Spiller, ibid. 78, 052326 (2008).

[2] S. J. Devitt, A. D. Greentree, R. Ionicioiu, J. L. O'Brien, W. J. Munro, and L. C. L. Hollenberg, Phys. Rev. A 76, 052312 (2007).

[3] T. B. Pittman, B. C. Jacobs, and J. D. Franson, Phys. Rev. A 64, 062311 (2001).

[4] K. Nemoto and W. J. Munro, Phys. Rev. Lett. 93, 250502 (2004).

[5] C. W. J. Beenakker, D. P. DiVincenzo, C. Emary, and M. Kindermann, Phys. Rev. Lett. 93, 020501 (2004).

[6] E. Knill, R. Laflamme, and G. J. Milburn, Nature (London) 409, 46 (2001).

[7] J. Eisert, Phys. Rev. Lett. 95, 040502 (2005).

[8] P. Kok, W. J. Munro, K. Nemoto, T. C. Ralph, J. P. Dowling, and G. J. Milburn, Rev. Mod. Phys. 79, 135 (2007).

[9] D. B. Uskov, L. Kaplan, A. M. Smith, S. D. Huver, and J. P. Dowling, Phys. Rev. A 79, 042326 (2009).

[10] A. J. F. Hayes, H. L. Haselgrove, A. Gilchrist, and T. C. Ralph, Phys. Rev. A 82, 022323 (2010).

[11] N. Imoto, H. A. Haus, and Y. Yamamoto, Phys. Rev. A 32, 2287 (1985).

[12] P. Grangier, J. A. Levenson, and J.-P. Poizat, Nature (London) 396, 537 (1998).

[13] L.-M. Duan, G. Giedke, J. I. Cirac, and P. Zoller, Phys. Rev. A 62, 032304 (2000).

[14] G. J. Milburn, Phys. Rev. Lett. 62, 2124 (1989).

[15] C. C. Gerry and R. Grobe, Phys. Rev. A 75, 034303 (2007).

[16] G. D. Hutchinson and G. J. Milburn, J. Mod. Opt. 51, 1211 (2004).

[17] D. Vitali, M. Fortunato, and P. Tombesi, Phys. Rev. Lett. 85, 445 (2000).

[18] P. Kok, H. Lee, and J. P. Dowling, Phys. Rev. A 66, 063814 (2002).

[19] N. Matsuda, R. Shimizu, Y. Mitsumori, H. Kosaka, and K. Edamatsu, Nature Photon. 3, 95 (2009).

[20] H. Jeong, Phys. Rev. A 73, 052320 (2006).

[21] P. Kok, Phys. Rev. A 77, 013808 (2008).

[22] H. Schmidt and A. Imamoğlu, Opt. Lett. 21, 1936 (1996).

[23] M. D. Lukin and A. Imamoğlu, Nature (London) 413, 273 (2001).

[24] T. Opatrný and D.-G. Welsch, Phys. Rev. A 64, 023805 (2001).

[25] H. Kang and Y. Zhu, Phys. Rev. Lett. 91, 093601 (2003).

[26] A. Joshi and M. Xiao, Phys. Rev. A 72, 062319 (2005).

[27] L. M. Kuang, Z. B. Chen, and J.-W. Pan, Phys. Rev. A 76, 052324 (2007); Y. Guo, S. S. L, and L. M. Kuang, J. Phys. B: At. Mol. Opt. Phys. 44, 065501 (2011).

[28] S. Li, X. Yang, X. Cao, C. Zhang, C. Xie, and H. Wang, Phys. Rev. Lett. 101, 073602 (2008).

[29] H. Sun, X. L. Feng, S. Gong, and C. H. Oh, Phys. Rev. B 79, 193404 (2009).

[30] H.-Y. Lo, P.-C. Su, and Y.-F. Chen, Phys. Rev. A 81, 053829 (2010).

[31] M. Fleischhauer, A. Imamoglu, and J. P. Marangos. Rev.
Mod. Phys. 77, 633 (2005).

[32] Q. Guo, J. Bai, L. Y. Cheng, X. Q. Shao, H. F. Wang, and S. Zhang, Phys. Rev. A 83, 054303 (2011).

[33] Q. Lin and J. Li Phys. Rev. A 79, 022301 (2009).

[34] S. D. Barrett and G. J. Milburn, Phys. Rev. A 74, 060302 (2006).

[35] W. J. Munro, K. Nemoto, and T. P. Spiller, New J. Phys. 7, 137 (2005).

[36] S. G. R. Louis, W. J. Munro, T. P. Spiller, and K. Nemoto, Phys. Rev. A 78, 022326 (2008).

[37] S. D. Barrett, P. Kok, K. Nemoto, R. G. Beausoleil, W. J. Munro, and T. P. Spiller, Phys. Rev. A 71, 060302(R) (2005).

[38] F. Dell'Anno, S. De Siena, and F. Illuminati, Phys. Rep. 428, 53 (2006).

[39] B. He, M. Nadeem, and J. A. Bergou, Phys. Rev. A 79, 035802 (2009).

[40] S. Ya. Kilin and A. B. Mikhalychev, Phys. Rev. A 83, 052303 (2011).

[41] C. C. Gerry, J. Mimih, and R. Birrittella, Phys. Rev. A 84, 023810 (2011).

[42] G. M. D’Ariano, L. Maccone, M. G. A. Paris, and M. F. Sacchi, Phys. Rev. A 61, 053817 (2000); Fortsch. Phys. 48, 671 (2000).

[43] M. G. A. Paris, J. Phys. B: At. Mol. Opt. Phys. 40, F63 (2007).

[44] R. L. Cook, P. J. Martin, and J. M. Geremia, Nature (London) 446, 774 (2007).

[45] C. Wittmann, U. L. Andersen, M. Takeoka, D. Sych, and G. Leuchs, Phys. Rev. Lett. 104, 100505 (2010); Phys. Rev. A 81, 062338 (2010).

[46] H. Zhang et al., Nature Photon. 5, 628 (2011).

[47] M. O. Scully and M. S. Zubairy, Quantum Optics (Cambridge University Press, Cambridge, England, 1997).

[48] D. Rosenberg, A. E. Lita, A. J. Miller, and S. W. Nam, Phys. Rev. A 71, 061803(R) (2005).

[49] O. Thomas, Z. L. Yuan, J. F. Dynes, A. W. Sharpe, and A. J. Shields, Appl. Phys. Lett. 97, 031102 (2010).

[50] W. H. P. Pernice, C. Schuck, O. Minaeva, M. Li, G. N. Goltsman, A. V. Sergienko, H. X. Tang, arXiv: 1108.5299v2 [quant-ph].

[51] Y. B. Sheng, F. G. Deng, and G. L. Long, Phys. Rev. A 82, 032318 (2010).

[52] S. G. R. Louis, K. Nemoto, W. J. Munro, and T. P. Spiller, Phys. Rev. A 75, 042323 (2007).

[53] S. J. van Enk and O. Hirota, Phys. Rev. A 64, 022313 (2001); S. J. van Enk, ibid. 72, 022308 (2005).

[54] G. F. Sinclair and N. Korolkova, Phys. Rev. A 77, 033843 (2008).

[55] B. He, Q. Lin, and C. Simon, Phys. Rev. A 83, 053826 (2011).

[56] J. H. Shapiro and M. Razavi, New J. Phys. 9, 16 (2007).

[57] P. M. Leung, T. C. Ralph, W. J. Munro, and K. Nemoto, arXiv: $0810.2828 \mathrm{v} 2$ [quant-ph].

[58] F. G. Deng, Phys. Rev. A 85, 022311 (2012). 